\section{Perspectives on Nuclear Medicine for Molecular Diagnosis and Integrated Therapy}

Y. Kuge, T. Shiga, and N. Tamaki, Eds.

Tokyo, Japan: Springer Japan, 2016, 326 pages, $\$ 59.99$

Professor Tamaki and his associates at Hokkaido University conducted international symposia on PET and molecular imaging in 1999, 2003, and 2009 and published the proceedings for each. This book is based on their 2015 international symposium entitled "Perspectives on Nuclear Medicine for Molecular Diagnosis and Integrated Therapy." The symposium, attended by more than 100 physicians and scientists, provided a chance to exchange ideas on how to promote nuclear medicine technology and how to extend that technology to medical diagnosis, therapy, and drug development. The symposium was also a good opportunity for worldwide experts in the field to discuss their perspectives on the future of nuclear medicine and molecular imaging.

Molecular imaging allows for the visualization of specific molecular and cellular targets that are relevant to tissue characterization, and the technologies of molecular imaging have rapidly developed in recent years. Among them, nuclear medicine technologies using PET and SPECT have come to play important roles in the quantitative analysis of biologic processes and now are widely used in clinical practice, especially for monitoring treatment efficacy by serial assessment of molecular function. Therapeutic response may be predicted only by using imaging with radiolabeled drugs. Therefore, a new era has arrived with the use of molecular imaging for personalized medicine.

This book was written by 99 contributors, mostly Japanese professors, and is organized into 5 parts with 25 chapters. Part 1, with 5 chapters, deals with instrument and data analysis, and part 2, with 6 chapters, discusses biomarker and molecular probes. Part 3 , with 5 chapters, handles applications of PET and SPECT in cardiology, and part 4 , with 4 chapters, deals with the utility of PET, SPECT, and MRI in neurology. Part 5, with 5 chapters, discusses PET applications in oncology, as well as the therapeutic roots of nuclear medicine and communication between PET specialists and oncologists. All 124 figures are in black and white but readable, and the 24 tables are instructive. The references are updated. There is no index.

This book will probably be beneficial to researchers in various fields of the life sciences, particularly those working in drug development or in molecular imaging and therapy, as well as specialists and trainees in nuclear medicine and radiology.

\author{
E. Edmund Kim \\ University of California at Irvine \\ 101 The City Dr. S. \\ Orange, CA 92868 \\ E-mail: edmundek@uci.edu
}

